Article

\title{
Biotransformation of Resveratrol: New Prenylated trans-Resveratrol Synthesized by Aspergillus sp. SCSIOW2
}

\author{
Liyan Wang ${ }^{1,2, *}$, Yanhua $\mathrm{Wu}^{1,2}$, Yongtao Chen ${ }^{1,2}$, Jiaxin Zou ${ }^{1,2}$ and Xiaofan $\mathrm{Li}^{1,2, *}$ \\ 1 Shenzhen Key Laboratory of Marine Bioresource and Eco-environmental Science, College of Life Sciences \\ and Oceanography, Shenzhen University, Shenzhen 518060, China; 13723776301@139.com (Y.W.); \\ cyto2011@126.com (Y.C.); 13691608828@163.com (J.Z.) \\ 2 Shenzhen Key Laboratory of Microbial Genetic Engineering, College of Life Sciences and Oceanography, \\ Shenzhen University, Shenzhen 518060, China \\ * Correspondence: lwang@szu.edu.cn (L.W.); lixiaof@szu.edu.cn (X.L.); \\ Tel.: +86-755-2601-2653 (L.W.); Fax: +86-755-2653-4274 (L.W.)
}

Academic Editor: Norbert Latruffe

Received: 7 June 2016; Accepted: 4 July 2016; Published: 6 July 2016

\begin{abstract}
Arahypin-16 (1), a new prenylated resveratrol with a unique dihydrobenzofuran ring, has been isolated as a microbial metabolite of resveratrol (2) from whole-cell fermentation of Aspergillus sp. SCSIOW2. The stereochemistry of $\mathbf{1}$ was determined by ECD calculations. 1 showed about half of the extracellular radical scavenging effect $\left(\mathrm{IC}_{50}=161.4 \mu \mathrm{M}\right)$ compared with resveratrol $\left(\mathrm{IC}_{50}=80.5 \mu \mathrm{M}\right)$, while on biomembranes it exhibited the same range of protection effects against free radicals generated from $\mathrm{AAPH}\left(\mathrm{IC}_{50}=78.6 \mu \mathrm{M}\right.$ and $\left.87.9 \mu \mathrm{M}\right)$.
\end{abstract}

Keywords: resveratrol; prenylation; Aspergillus sp. SCSIOW2; ECD; erythrocyte protection activity; DPPH scavenging activity

\section{Introduction}

Resveratrol is well known for the potential effects on coronary heart disease observed among wine drinkers [1]. Nowadays resveratrol, which possesses a wide range of biological activities, such as antioxidant, anti-inflammatory, antiviral, antimicrobial, anticancer, cardiovascular protection, chemo-protection, neuro-protection and immune-modulation activity is one of the best studied natural products [2]. Previous data have provided interesting insights into the effects of this compound on the lifespan of different organisms and it might become a potential anti-aging agent in degenerative human diseases [3]. However, any further medicinal application of resveratrol is limited due to its low bioavailability and solubility. Researchers have found the addition of isoprenoid substituents on various polyphenol skeletons significantly increased the bioactivity compared to similar compounds that are not prenylated [4]. For example, the introduction of an 8-prenyl group induces estrogenic activity in naringenin. Additionally, 8-prenylnaringenin is rapidly absorbed after oral administration, compared with the generally poor oral bioavailability of flavonoids [5]. Therefore, different strategies have been developed for synthesis of such compounds. The most commonly used organic synthetic strategies are either activated by strong base or by coupling reactions catalyzed by metal salts. Both reactions are usually carried out under extreme conditions and additional steps protection and deprotection are needed. The chemoselectivity, regiochemistry, and number of prenyls are all also difficult to control [6]. Recently, chemo-enzymatic syntheses using enzymes or whole cells were also used for the synthesis of prenylated aromatic components. This type reactions are usually regioselective, stereoselective and occur under mild conditions, thus the introduction of prenyl groups into target compounds by the use of microorganisms represents an attractive alternative to conventional 
chemical synthesis $[7,8]$. A series of prenylated resveratrols which have not been previously reported from peanuts has been isolated from peanut seeds challenged by different fungi. These new compounds were considered to play a defensive role against invasive fungi. However, the bioactivities and the real origin of these components were not fully discussed [9-11].

We are interested in utilizing microbial cultures as biocatalysts to prepare new and potentially active analogues of polyphenolic compounds. In this study, Aspergillus sp. SCSIOW2 showed high activity for the regiospecific prenylation of resveratrol. Here we describe the biotranformation, isolation and structure elucidation of arahypin-16 (1), a new prenylated resveratrol with an unique dihydrobenzofuran ring, as a microbial metabolite of resveratrol (2) from whole-cell fermentation of Aspergillus sp. SCSIOW2 (Figure 1).
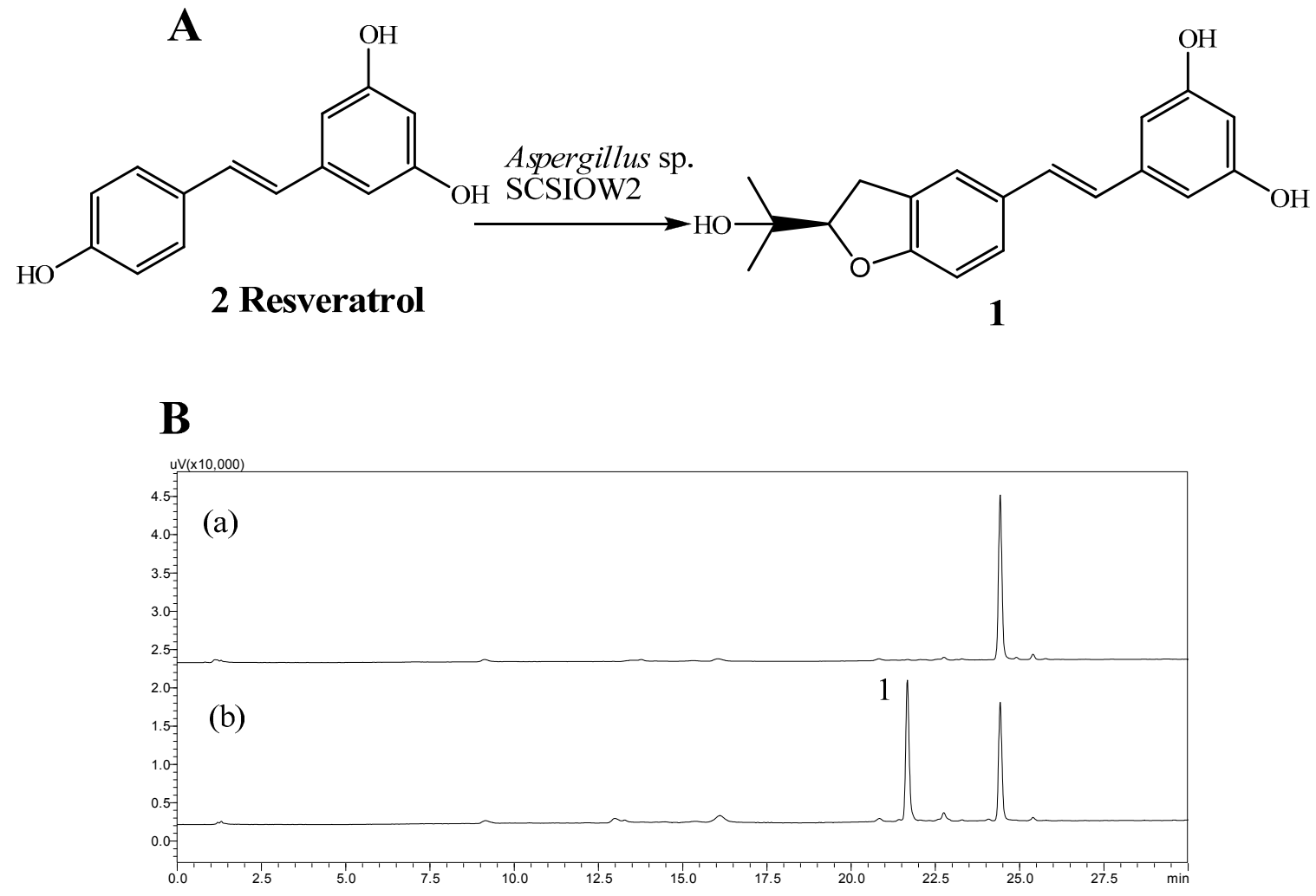

Figure 1. The conversion of trans-resveratrol into $\mathbf{1}$ is catalyzed by Aspergillus sp. SCSIOW2. (A): Structures of trans-resveratrol (2) and arahypin-16 (1); (B): HPLC-UV chromatogram at $309 \mathrm{~nm}$ of EtOAc extracts of untreated (a) and resveratrol-treated (b) fermentation broth.

\section{Results and Discussions}

A total of 21 fungi strains were screened for their ability to biotransform resveratrol. On the basis of comparative HPLC analyses of test cultures and controls, Aspergillus sp. SCSIOW2 was the only organism capable of biotransforming 2 into new metabolites (Figure 1). A preparative scale biotransformation of $\mathbf{2}$ using whole-cell fermentation afforded metabolite $\mathbf{1}$.

Arahypin-16 (1) was isolated as a pale white amorphous powder. The LC-ESI-MS showed a $[\mathrm{M}+\mathrm{Na}]^{+}$peak at 335. Using HRESIMS, a molecular ion was measured at 391.0556 (calcd. for $\mathrm{C}_{19} \mathrm{H}_{20} \mathrm{BrO}_{4}[\mathrm{M}+\mathrm{Br}]^{-}$, 391.0550), indicating a molecular formula of $\mathrm{C}_{19} \mathrm{H}_{20} \mathrm{O}_{4}$ with 10 degrees of unsaturation. The ${ }^{1} \mathrm{H}$ - and ${ }^{13} \mathrm{C}-\mathrm{NMR}$ spectra of 1 were similar to those of the previously isolated stilbene arahypin-2, which contained a resveratrol skeleton with a dioxygenated prenyl group at the C-2 position [11]. The ${ }^{1} \mathrm{H}-\mathrm{NMR}$ spectrum of 1 showed a triplet at $\delta_{\mathrm{H}} 6.11$ and a doublet at $\delta_{\mathrm{H}} 6.38$ $(2 \mathrm{H})$ of an $\mathrm{AB} 2$ system for ring $\mathrm{B}$; a singlet at $\delta_{\mathrm{H}} 7.44$, a doublet at $\delta_{\mathrm{H}} 7.24$, and a doublet at $\delta_{\mathrm{H}} 6.71$ for 
ring A; two coupled doublets at $\delta_{\mathrm{H}} 6.94$ and 6.83 for a trans double bond; and a dioxygenated prenyl group (Table 1). However, the mass of 1 was found to be 18 units less than arahypin-2, corresponding to a difference of a $\mathrm{H}_{2} \mathrm{O}$ molecule. Using DMSO- $d_{6}$ as the solvent allowed also assignments of three hydroxyl protons of $\mathbf{1}$ (Table 1). Thus, the difference between $\mathbf{1}$ and arahypin-2 could be the cyclization of the prenyl unit with the oxygen at 16 or 17 position on the 1,2,4-trisubstituted ring. The dihydrobenzofuran moiety was finally established by HMBC correlations from $\mathrm{H}-16$ to $\mathrm{C}-1$ and from 17-OH to Me-18 and Me-19 (Table 1, Figure 2). Finally, careful ${ }^{1} \mathrm{H}-{ }^{1} \mathrm{H}$ COSY, HMQC, and HMBC analyses confirmed the structure as $\mathbf{1}$ (Table 1, Figure 2).

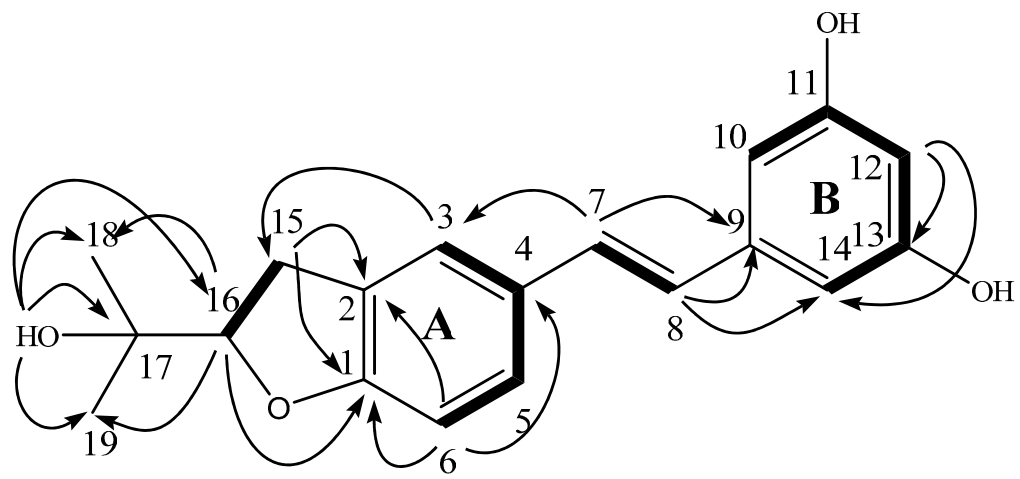

Figure 2. Key ${ }^{1} \mathrm{H}-{ }^{1} \mathrm{H}$ COSY and HMBC correlations of $\mathbf{1}$ and 2.

Table 1. NMR spectroscopic data for compound $1\left(\mathrm{DMSO}-d_{6}\right)^{\mathrm{a}}$.

\begin{tabular}{|c|c|c|c|}
\hline Position & $\delta_{H}(\text { Mult, } J \text { in } \mathrm{Hz})^{b}$ & $\delta_{C}^{c}$ & HMBC \\
\hline 1 & & 160.1 & \\
\hline 2 & & 128.7 & \\
\hline 3 & $7.44 \mathrm{~s}$ & 123.0 & $C-1,5,15$ \\
\hline 4 & & 129.8 & \\
\hline 5 & $7.24 \mathrm{~d}(8.4)$ & 127.4 & $C-1,3,7$ \\
\hline 6 & $6.71 \mathrm{~d}(8.4)$ & 109.1 & C- $-1,2,4$ \\
\hline 7 & $6.94 \mathrm{~d}(16.2)$ & 128.4 & $C-3,5,9$ \\
\hline 8 & $6.83 \mathrm{~d}(16.2)$ & 126.2 & C- $4,7,9,10,14$ \\
\hline 9 & & 139.7 & \\
\hline $10 / 14$ & $6.38 \mathrm{~d}(1.8) 2 \mathrm{H}$ & 104.7 & $C-8,11,12$ \\
\hline 11 & & 158.9 & \\
\hline 12 & $6.11 \mathrm{t}(1.8)$ & 102.2 & C- $10,11,13,14$ \\
\hline 13 & & 158.9 & \\
\hline 15 & $3.13 \mathrm{~m}$ & 30.2 & $C-1,2,3,16,17$ \\
\hline 16 & $4.56 \mathrm{t}(9.0)$ & 89.7 & $\mathrm{C}-1,2,18,19$ \\
\hline 17 & & 70.5 & \\
\hline 18 & $1.14 \mathrm{~s}^{\mathrm{e}}$ & $25.2^{\mathrm{e}}$ & C-16, 17, 19 \\
\hline 19 & $1.12 \mathrm{~s}^{\mathrm{e}}$ & $26.5^{\mathrm{e}}$ & $\mathrm{C}-16,17,18$ \\
\hline $11-\mathrm{OH}$ & $9.22 \mathrm{~s} \mathrm{~d}$ & & \\
\hline $13-\mathrm{OH}$ & $9.22 \mathrm{~s} \mathrm{~d}$ & & \\
\hline $17-\mathrm{OH}$ & $4.61 \mathrm{~s}$ & & C-17, 18, 19 \\
\hline
\end{tabular}

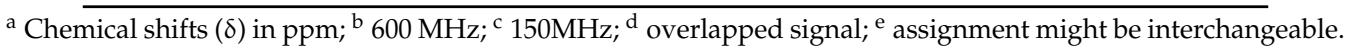

The ECD spectrum of $\mathbf{1}$ was then recorded and compared with those calculated for each enantiomer using the time-dependent density functional theory (TDDFT) method [12-17]. After conformation space analysis, 22 conformers were found for $\mathbf{1}$ (Table S1 shows the equilibrium populations of 22 stable conformations in methanol at the B3LYP/aug-cc-pVDZ level). Consequently, the calculated ECD spectrum for the enantiomer $16 R$ fit with the experimental plot of $\mathbf{1}$, which exhibited two negative and two positive Cotton effects at 315, 245, 263, and $221 \mathrm{~nm}$, respectively. However, 
the calculated ECD spectrum of the 16-(S) enantiomer was opposite to the experimental ECD data (Figure 3). Hence, the stereochemistry of 1 was determined to be 16-(R).

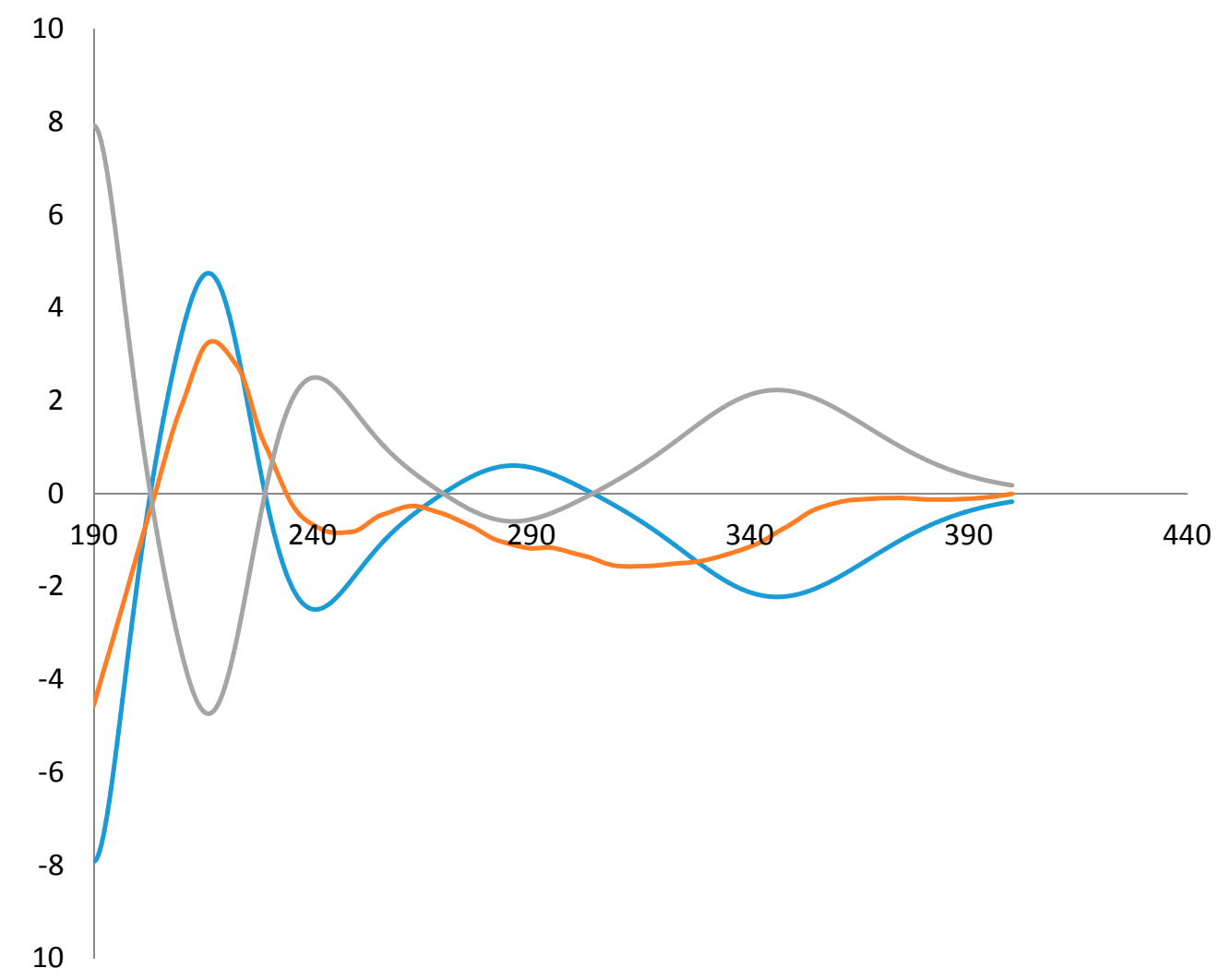

Figure 3. Comparison of the experimental ECD spectrum of 1 (red) with those calculated for the enantiomers 16-(R) (blue) and 16-(S) (green). (UV correction $=0 \mathrm{~nm}$, bandwidth $\sigma=0.16 \mathrm{eV}$ ).

Oxidative damages of cell membranes is closely connected with various free radical-mediated diseases such as rheumatoid arthritis, atherosclerosis, cancer, etc. [18]. Hemolysis of erythrocytes is a relatively simple model for studying oxidative damage to biomembranes due to its enucleated composition [19]. The antioxidant activity of our compounds was evaluated through biomembrane protection against hemolysis induced by 2,2 '-azobis(2-methylpropionamidine) dihydrochloride (AAPH), with $\mathrm{IC}_{50}$ values of $78.6 \mu \mathrm{M}$ of 1 and $87.9 \mu \mathrm{M}$ of 2 , respectively (Figure 4 ). The DPPH scavenging activity was also assayed to clarify their capacity for scavenging extracellular free radicals, affording $\mathrm{IC}_{50}$ values of $161.4 \mu \mathrm{M}$ of $\mathbf{1}$ and $80.5 \mu \mathrm{M}$ of $\mathbf{2}$, respectively (Figure 5). Comparing the two experiments, the prenylated compound arahypin-16 (1) showed about half the extracellular radical scavenging of resveratrol, while it exhibited the same range of biomembrane protection effect against free radicals produced by AAPH.

Prenylated components are widely distributed in natural products and exhibit a wide range of biological activities. However, in most cases it is not clear whether the prenyl group has a specific role in the bioactivity. The shortage of systematic studies on prenylated components is mainly due to their limited availability. Recently, an impressive number of bioactivities have been demonstrated for prenylated flavonoids, especially for cancer chemotherapy, which has inspired researchers to generate new prenylated components by using different strategies [20]. Chemo-enzymatic syntheses have drawn the attention of scientists, because they are mostly regioselective and stereoselective and occur under mild conditions. Prenyltransferases have been successfully used for the production of more than 250 prenylated derivatives, such as prenylated tyrosine, xanthone, hydroxynaphthalenes, flavonoids, indolocarbazoles, and acylphloroglucinols [6]. In this study, Aspergillus sp. SCSIOW2 showed high 
regiospecific prenylation activity with resveratrol, generating arahypin-16 (1), a new component with an unique dihydrobenzofuran ring. This is the first report about the prenylation of resveratrol by fungal biotransformation, which provided us a prenylated resveratrol, arahypin-16 (1), for further bioactivity comparison.
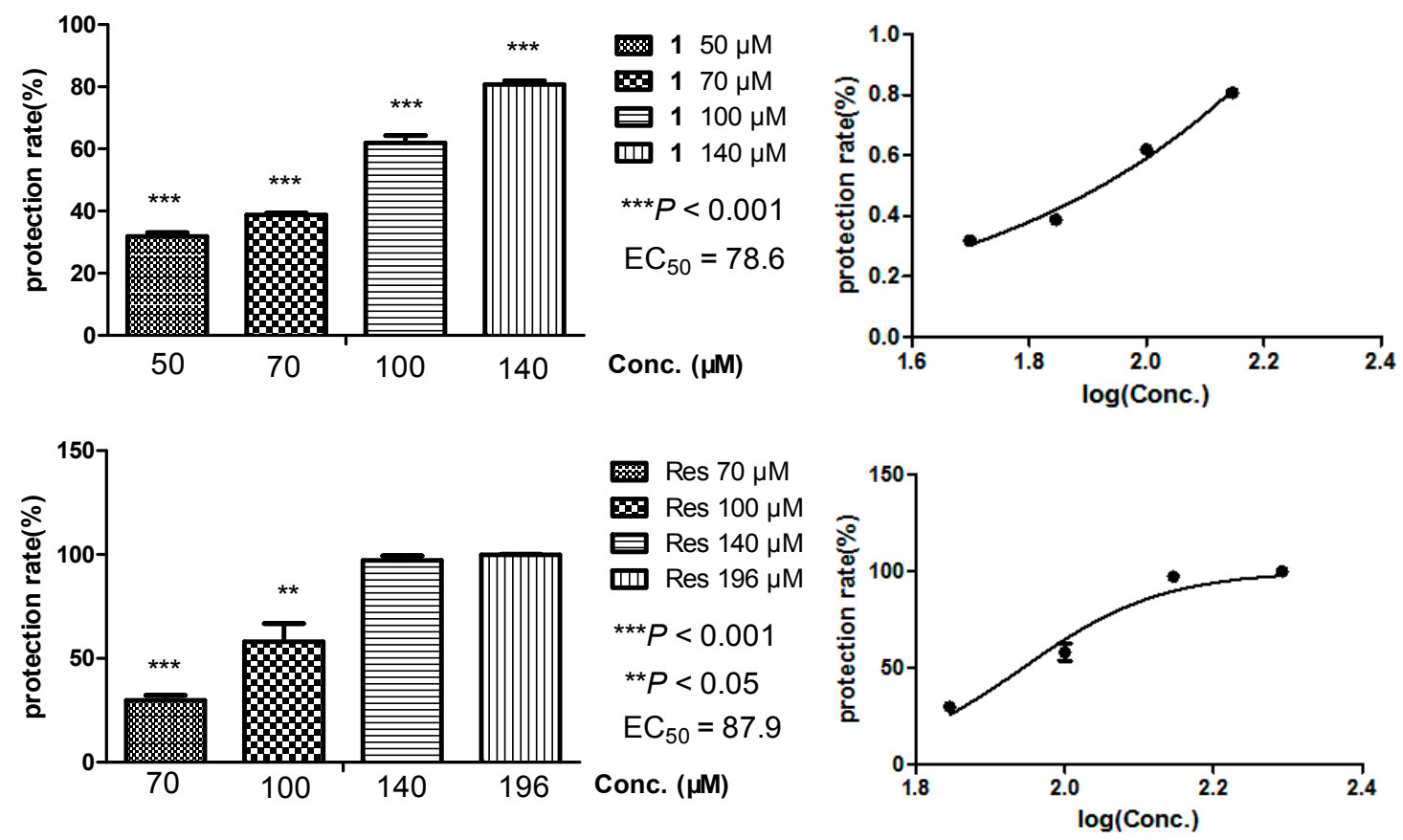

Figure 4. Erythrocyte membrane protection rate (\%) of arahypin-16 (1) and resveratrol (Res) against hemolysis induced by AAPH.
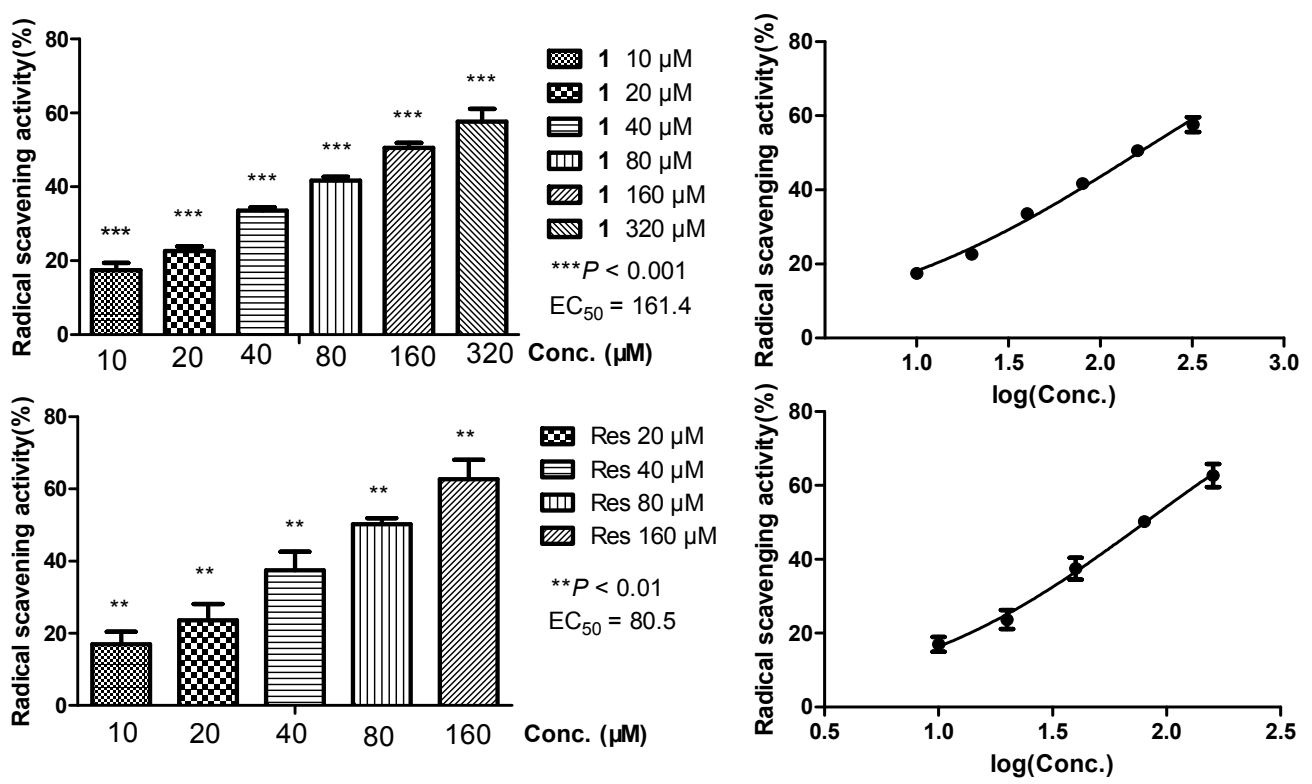

Figure 5. DPPH radical scavenging activity (\%) of arahypin-16 (1) and resveratrol (Res). 


\section{Materials and Methods}

\subsection{General Procedures}

Optical rotations were determined on a P-1020 polarimeter (Jasco, Tokyo, Japan). UV data were recorded on a Lambda $25 \mathrm{UV} / \mathrm{V}$ is spectrometer (Perkin Elmer, Boston, MA, USA). IR data were recorded using a Nicolet Avatar 330 FT-IR spectrometer (Thermo Scientific, Waltham, MA, USA). NMR spectra were acquired on a Bruker ASCEND $600 \mathrm{MHz}$ NMR magnet system (Bruker, Ettlingen, Germany) using TMS as the internal standard. HPLC-ESIMS was performed using a 6120 Single Quad LC/MS system (Agilent Technologies, Santa Clara, CA, USA). HR-ESIMS was performed using an Bruker maXis). CD spectra were recorded on a Jasco J-815 CD spectrometer. Column chromatography was conducted using silica gel (100-200 mesh, Qingdao Marine Chemical Factory, Qingdao, China) and Sephadex LH-20 (Amersham Pharmacia Biotech, Piscataway, NJ, USA). TLC was performed on Merck TLC plates (silica gel 60 RP-18 F254S and silica gel 60 F254, Merck Millipore Corporation, Darmstadt, Germany), with compounds visualized by spraying with $10 \%(v / v) \mathrm{H}_{2} \mathrm{SO}_{4}$ in $\mathrm{EtOH}$ and then heating on a hot plate. HPLC was performed on a Shimadzu LC-20AT pump (Shimadzu Corporation, Tokyo, Japan) equipped with a SPD-20A UV-Vis detector and an Agilent Technologies 1260 Infinity series with a 1260 DAD detector. An Agilent Technologies ZORBAX RX-C18 analytical column $(4.6 \times 150$ mm, $5 \mu)$ was used for analysis and semi-preparative purposes. Resveratrol was purchased from Sinopharm Chemical Reagent Co., Ltd. (Shanghai, China). The experimental New Zealand rabbit was purchased from Guangdong Medical Laboratory Animal Center (animal license: SCXK (Guangdong Province) 2014-0035) and fed at $22 \pm 2{ }^{\circ} \mathrm{C}$ with relative humidity at 50\%. The absorbance measurement in erythrocyte protection assay and DPPH scavenging assay was performed with Varios-Kan Flash (Thermo Fisher Scientific).

\subsection{Strain}

Fungus SCSIOW2 was isolated from a deep marine sediment sample collected in the South China Sea $\left(112^{\circ} 30.203 \mathrm{E}, 18^{\circ} 1.654 \mathrm{~N}\right)$ at a depth of $2439 \mathrm{~m}$. This fungus was characterized as Aspergillus sp. Based on the analysis of the ITS region sequence with Genebank S1. This fungus was deposited in the Marine Microbial Lab., College of Life Science, Shenzhen University (Shenzhen, China).

\subsection{Fermentation, Extraction, and Isolation}

Seed medium $\left(10.0 \%\right.$ glucose, $2.0 \%$ soybean meal, $0.5 \%$ peptone, $0.2 \% \mathrm{NaCl}, 0.05 \% \mathrm{KH}_{2} \mathrm{PO}_{4}$, $0.024 \% \mathrm{MgSO}_{4}$, with the $\mathrm{pH}$ adjusted to 7.0$)$. Production medium (7.6\% sucrose, $2.6 \%$ soybean meal, $1.0 \% \mathrm{NaOAc}, 1.0 \%$ citric acid, $0.6 \% \mathrm{CaCO}_{3}, 0.5 \%$ glycerin, $0.01 \% \mathrm{MgSO}_{4}$, with the pH adjusted to 7.0 ). Aspergillus sp. SCSIOW2 was cultured in 250-mL Erlenmeyer flasks containing $50 \mathrm{~mL}$ of seed medium. After growing at $28^{\circ} \mathrm{C}, 220 \mathrm{rpm}$ for 3 days, the cellular material was placed in a sterile Falcon tube and mixed by vortexing for several minutes to create a uniform fungal cell/spore suspension. Aliquots $(1 \mathrm{~mL})$ of seed cultures were inoculated into $70 \mathrm{~mL}$ of production medium in $250-\mathrm{mL}$ Erlenmeyer flasks. At the time of inoculation, $0.5-\mathrm{mL}$ aliquot of EtOH-dissolved resveratrol $(16.0 \mathrm{mg})$ was added, resulting in final concentrations in the liquid media containing $1 \mathrm{mM}$ resveratrol. Control group was added with the same amount of $\mathrm{EtOH}$. The resulting cultures were fermented at $28^{\circ} \mathrm{C}$ under static conditions for 7 days. The fermented broth was filtered to obtain supernatant and mycelia. The supernatant broth was extracted three times with $70 \mathrm{~mL}$ of EtOAc. The EtOAc extracts of each condition were analyzed by reversed-phase HPLC on a ZORBAX RX-C18 analytical column $(4.6 \times 150 \mathrm{~mm}, 5 \mu)$ eluted with $\mathrm{MeOH}-\mathrm{H}_{2} \mathrm{O}$ (0:100-100:0 over $\left.30 \mathrm{~min}, 1.0 \mathrm{~mL} / \mathrm{min}\right)$. For preparative scale up, Aspergillus terreus was cultivated using $45250-\mathrm{mL}$ Erlenmeyer flasks containing $70 \mathrm{~mL}$ of production medium in the presence of $1 \mathrm{mM}$ resveratrol. The combined EtOAc extract after evaporation (580.0 $\mathrm{mg})$ was applied to a Sephadex LH-20 column chromatograph (CC) with $\mathrm{CHCl}_{3}-\mathrm{MeOH}$ (1:1) to afford seven fractions (Fr.1-Fr.7). Fr.4 (28.6 mg) was further purified by HPLC with a ZORBAX RX-C18 column eluted with $\mathrm{MeOH}-\mathrm{H}_{2} \mathrm{O}(0: 100-100: 0$ over $30 \mathrm{~min}, 1.0 \mathrm{~mL} / \mathrm{min})$ to yield compound $1\left(9.8 \mathrm{mg}, \mathrm{t}_{\mathrm{R}} 19.8 \mathrm{~min}\right)$. 
Arahypin-16 (1): white powder; $[\alpha]_{\mathrm{D}}^{27}+125^{\circ}(c$ 1.0, $\mathrm{MeOH})$; UV (MeOH) $\lambda \max (\log \varepsilon) 308$ (3.99) nm, 320 (3.98), 218 (3.97), 201 (4.09) nm; IR (film) $v_{\max }$ : 3251, 2976, 1594, 1491, 862.7, $833.1 \mathrm{~cm}^{-1}$; HRESIMS $\mathrm{m} / \mathrm{z} 391.0556[\mathrm{M}+\mathrm{Br}]^{-}$(Calcd. for $\left.\mathrm{C}_{19} \mathrm{H}_{20} \mathrm{BrO}_{4}, 391.0550\right) ;{ }^{1} \mathrm{H}$ - and ${ }^{13} \mathrm{C}-\mathrm{NMR}$ see Table 1 .

\subsection{Quantum Chemical ECD Calculations}

In this study, we used the ECD calculation protocol proposed by Nugroho and Morita [13]. The initial 3D structures of the molecules were prepared using Chem3D and minimized with the MMFF94S force field implemented in Chem3D. After the initial structure was further optimized with XedMin in default mode, the conformation space was sampled using XedeX with an energy window of $5 \mathrm{kcal} \cdot \mathrm{mol}^{-1}$ above the ground state and RMSD 0.8 to remove duplicated conformers [14]. Then, each conformer was optimized and verified as true minima of the potential energy surface using Gaussian 09 with the DFT method at the B3LYP/aug-cc-pVDZ level [15]. The polarizable continuum model (IEFPCM) was used to take the solvent effects of methanol into account. The optimized conformers were further used to perform a TDDFT calculation at the B3LYP/aug-cc-pVDZ level with the polarizable-conductor calculation model (IEFPCM, methanol as the solvent). In each TDDFT calculation, the 100 lowest electronic transitions were calculated for each conformer. The ECD spectra and overall ECD spectra (weighted by Boltzmann statistics) and comparison of the experimental and calculated spectra were performed using the software SpecDis $[16,17]$.

\subsection{Preparation of Erythrocytes}

Packed erythrocytes were obtained by centrifuging whole rabbit blood with $3.2 \%$ citrate at $700 \mathrm{~g}$ for $10 \mathrm{~min}$ at $4{ }^{\circ} \mathrm{C}$. Cells were washed three times with $0.9 \% \mathrm{NaCl}$ solution and finally suspended in the buffer solution to obtain a citrated blood, stored at $4{ }^{\circ} \mathrm{C}$ and were used within $48 \mathrm{~h}$.

\subsection{Measurement of Hemolysis and Erythrocytes Membrane Protection}

For the hemolysis assay, a method slightly modified from that of Niki, Etsuo, et al. was followed [12,13,21]. Briefly, $6 \times 10^{8} \mathrm{~mL}^{-1}$ of the washed erythrocytes were pre-warmed at $37^{\circ} \mathrm{C}$ and mixed with the assayed compounds dissolved in DMSO and diluted with $0.9 \% \mathrm{NaCl}$ solution to make final concentrations as described at Figure 4. After incubation at $37^{\circ} \mathrm{C}$ for $30 \mathrm{~min}, 250 \mu \mathrm{L}$ of AAPH solution at $100 \mathrm{mM}$ was added to induce hemolysis for $60 \mathrm{~min}$ at $37^{\circ} \mathrm{C}$. The reaction mixture was centrifuged at $700 \mathrm{~g}$ for $2 \mathrm{~min}$ and the absorbance of the supernatant was measured at $545 \mathrm{~nm}$. The relative hemolysis was calculated in comparison with the hemolysis in dd water, which was taken as $100 \%$. Each set of experiments was performed in triplicate.

\subsection{DPPH Radical Scavenging Activity}

DPPH scavenging assay was performed as described previously [22]. The assayed compounds were dissolved in DMSO and diluted with mixture of ethanol and 0.4 M HOAc/NaOAc buffer (3:1) to working concentrations, followed with the addition of 20\% $(\mathrm{m} / \mathrm{v})$ DPPH ethanol solution, and then incubated in dark for $30 \mathrm{~min}$. The absorbance at $517 \mathrm{~nm}$ was measured. DPPH scavenging activity of the samples was calculated in comparison with the absorbance in ethanol and HOAc/NaOAc buffer, which was taken as $100 \%$. All the experiments were carried out in triplicate.

\subsection{Statistical Analysis}

All results were expressed as mean \pm SD. Statistical significance ( $p$ values) of the results was calculated by Student's $t$ test using GraphPad Prism 5 software package (San Diego, CA, USA). The results were considered to be significant when $p<0.05$. 


\section{Conclusions}

In summary, we have reported the conversion of trans-resveratrol to arahypin-16 (1) catalyzed by Aspergillus sp. SCSIOW2. This is the first trans-resveratrol derivative containing a dihydrobenzofuran ring and the first report concerning prenylation of resveratrol by direct fungal biotransformation. Arahypin-16 (1) showed about half the extracellular radical scavenging of resveratrol, while it exhibited the same range of protective effect on biomembranes against free radicals produced by AAPH. This is also the first report on the biomembrane protection activities of resveratrol and its derivatives.

Supplementary Materials: The following are available online at http://www.mdpi.com/1420-3049/21/7/883/ s1, Table S1: Conformer distribution of 1 in solvated models (methanol) calculation at the B3LYP/aug-cc-PVDZ level, Figures S1-S6: NMR spectra for compound 1. Figure S1: ${ }^{1} \mathrm{H}-\mathrm{NMR}$ spectrum of $\mathbf{1}$ in DMSO-d $\mathrm{d}_{6}$, Figure S2: ${ }^{13} \mathrm{C}-\mathrm{NMR}$ spectrum and DEPT of 1 in DMSO- $d_{6}$, Figure S3: ${ }^{1} \mathrm{H}-{ }^{1} \mathrm{H}$ COSY spectrum of 1 in DMSO-d $\mathrm{d}_{6}$, Figure S4: HSQC spectrum of 1 in DMSO- $d_{6}$, Figure S5: HMBC spectrum of 1 in DMSO- $d_{6}$, Figure S6: NOESY spectrum of 1 in DMSO- $d_{6}$.

Acknowledgments: This work was supported by the National Natural Science Foundation of China (NSFC) under Grant number 41276136; the Science and Technology Project of Shenzhen City, Shenzhen Bureau of Science, Technology and Information under Grant JCYJ20150827101359276; and by Interdisciplinary Innovation Team Project of Shenzhen University. Mr. Gaokeng Xiao from Guangzhou Molcalx Information \& Technology Ltd. supported the ECD calculations.

Author Contributions: Liyan Wang, Yanhua Wu and Yongtao Chen conducted experiments. Jiaxin Zou conducted part of the fermentation and isolation. Liyan Wang analyzed the spectroscopic data and elucidated the structures. Liyan Wang wrote the manuscript; Xiaofan Li conducted the erythrocytes membrane protection and DPPH radical scavenging activty. Liyan Wang and Xiaofan Li supervised the research work.

Conflicts of Interest: The authors declare no conflict of interest.

\section{References}

1. Frankel, E.N.; Waterhouse, A.L.; Kinsella, J.E. Inhibition of human LDL oxidation by resveratrol. Lancet 1993, 341, 1103-1104. [CrossRef]

2. Liu, Y.; Liu, Y.; Chen, H.; Yao, X.; Xiao, Y.; Zeng, X.; Zheng, Q.; Wei, Y.; Song, C.; Zhang, Y.; et al. Synthetic resveratrol derivatives and their biological activities: A review. Open J. Med. Chem. 2015, 5, 97-105. [CrossRef]

3. Lanc, A.; Kaminski, J.; Tili, E. Control of microRNA expression as a new way for resveratrol to deliver its beneficial effects. J. Agric. Food Chem. 2012, 60, 8783-8789. [CrossRef] [PubMed]

4. Siregar, T.M.; Cahyana, H.; Wibowo, W. Application of superbase catalyst $\gamma-\mathrm{Al}_{2} \mathrm{O}_{3} / \mathrm{NaOH} / \mathrm{Na}$ for prenylation reaction of resveratrol (3,5,4'-trihydroxystilbene). J. Chem. Pharm. Res. 2014, 6, 349-353.

5. Rad, M.; Huemperl, M.; Schaefer, O.; Schoemaker, R.C.; Schleuniing, W.D.; Cohen, A.F. Pharmacokinetics and systemic endocrine effects of the phyto-oestrogen 8-prenylnaringenin after single oral doses to postmenopausal women. J. Br. J. Clin. Pharmacol. 2006, 62, 288-296. [CrossRef] [PubMed]

6. Fan, A.; Winkelblech, J.; Li, S. Impacts and perspectives of prenyltransferases of the DMATS superfamily for use in biotechnology. Appl. Microbiol. Biot. 2015, 99, 7399-7415. [CrossRef] [PubMed]

7. Okamoto, R.; Izumi, M.; Kajihara, Y. Decoration of proteins with sugar chains: recent advances in glycoprotein synthesis. Curr. Opin. Chem. Biol. 2014, 22, 92-99. [CrossRef] [PubMed]

8. Yu, X.; Liu, Y.; Xie, X.; Zheng, X.; Li, S. Biochemical characterization of indole prenyltransferases: Filling the last gap of prenylation positions by a 5-dimethylallyltryptophan synthase from Aspergillus clavatus. J. Biol. Chem. 2012, 287, 1371-1380. [CrossRef] [PubMed]

9. Sobolev, V.S.; Krausert, N.M.; Gloer, J.B. New Monomeric stilbenoids from peanut (Arachis hypogaea) seeds challenged by an Aspergillus flavus strain. J. Agric. Food Chem. 2016, 64, 579-584. [CrossRef] [PubMed]

10. Aisyah, S.; Gruppen, H.; Slager, M.; Helmink, B.; Vincken, J. Modification of prenylated stilbenoids in peanut (Arachis hypogaea) seedlings by the same Fungi that elicited them: the Fungus strikes back. J. Agric. Food Chem. 2015, 63, 9260-9268. [CrossRef] [PubMed]

11. Sobolev, V.S.; Neff, S.A.; Gloer, J.B. New dimeric stilbenoids from fungal-challenged peanut (Arachis hypogaea) seeds. J. Agric. Food Chem. 2010, 58, 875-881. [CrossRef] [PubMed]

12. Yan, S.L.; Su, Y.F.; Chen, L.; Que, M.; Gao, X.M.; Chang, J.B. Polygonumosides A-D, stilbene derivatives from processed roots of Polygonum multiflorum. J. Nat. Prod. 2014, 77, 397-401. [CrossRef] [PubMed] 
13. Nugroho, E.A.; Morita, H. Circular dichroism calculation for natural products. J. Nat. Med. 2014, 68, 1-10. [CrossRef] [PubMed]

14. XedeX. Available online: http://www.cresset-group.com (accessed on 5 July 2015).

15. Frisch, M.J.; Trucks, G.W.; Schlegel, H.B.; Scuseria, G.E.; Robb, M.A.; Cheeseman, J.R.; Scalmani, G.; Barone, V.; Mennucci, B.; Petersson, G.A.; et al. Gaussian 09, Revision D. 01; Gaussian Inc.: Wallingford, CT, USA, 2013.

16. Bruhn, T.; Schaumlöffel, A.; Hemberger, Y.; Bringmann, G. SpecDis, Version 1.60; University of Wuerzburg: Wuerzburg, Germany, 2013.

17. Bruhn, T.; Schaumlöffel, A.; Hemberger, Y.; Bringmann, G. SpecDis: Quantifying the comparison of calculated and experimental electronic circular dichroism spectra. Chirality 2013, 25, 243-249. [CrossRef] [PubMed]

18. Chaudhuri, S.; Banerjee, A.; Basu, K.; Sengupta, B.; Sengupta, P.K. Interaction of flavonoids with red blood cell membrane lipids and proteins: antioxidant and antihemolytic effects. Int. J. Boil. Macromol. 2007, 41, 42-48. [CrossRef] [PubMed]

19. Banerjee, A.; Kunwar, A.; Mishra, B.; Priyadarsini, K.I. Concentration dependent antioxidant/pro-oxidant activity of curcumin studies from AAPH induced hemolysis of RBCs. Chemico-Biol. Interact. 2008, 174, 134-139. [CrossRef] [PubMed]

20. Bruno, B.; Giuliano, D.M.; Pilar, M.; Alberto, B. Novel prenyltransferase enzymes as a tool for flavonoid prenylation. TRENDS Pharmacol. Sci. 2005, 26, 606-608.

21. Niki, E.; Komuro, E.; Takahashi, M.; Urano, S.; Ito, E.; Terao, K. Oxidative hemolysis of erythrocytes and its inhibition by free radical scavengers. J. Boil. Chem. 1988, 263, 19809-19814.

22. Li, X.; Li, L.; Wang, J.; Wang, T.; Wang, L. Two new lignans with antioxidative activities from Jatropha curcas. Nat. Prod. Res. 2014, 28, 1985-1991. [CrossRef] [PubMed]

Samples Availability: Samples of the compounds $\mathbf{1}$ and $\mathbf{2}$ are available from the authors.

(C) 2016 by the authors; licensee MDPI, Basel, Switzerland. This article is an open access article distributed under the terms and conditions of the Creative Commons Attribution (CC-BY) license (http://creativecommons.org/licenses/by/4.0/). 